\title{
Artificial Neural Network Context-Aware Reasoning Engine Model for Predicting User-Ranked Activities
}

\author{
Samuel King Opoku
}

\begin{abstract}
The choice of users' activity in a context-aware environment depends on users' preferences and background. Users tend to rank concurrent activities and select their preferred activity. Researchers and developers of context-aware applications have sought various mechanisms to implement context reasoning engines. Recent implementations use Artificial Neural Networks (ANN) and other machine learning techniques to develop a context-aware reasoning engine to predict users' activities. However, the complexities of these mechanisms overwhelm the processing capabilities and storage capacity of mobile devices. The study models a context-aware reasoning engine using a multi-layered perceptron with a gradient descent back-propagation algorithm to predict activity from user-ranked activities using a stochastic learning mode with a constant learning rate. The work deduced that working with specific rules in training a neural network is not always applicable. Training a network without approximation of neuron's output to the nearest whole number increases the accuracy level of the network at the end of the training.
\end{abstract}

Keywords - Activity Context, Artificial Neural Network, Backpropagation, Context-Aware, Reasoning Engine.

\section{INTRODUCTION}

The ability to design an intelligent reasoning engine for context-aware mobile applications has prompted many researchers to devise various mechanisms. Previous mechanisms included if-then rules, key-value, logic-based and ontology-based models [1]. Following the use of ontology-based models complemented with other technologies in acquiring activity context, Dey [2], Naeem et al. [3] and Sherif and Alesheikh [4] suggested the need to use machine learning approach in designing context-aware applications. Two branches of Artificial Intelligence that support machine learning are Evolutionary Computing and Artificial Neural Network (ANN) (Banerjee, 2015). Evolutionary Computing involves working with algorithms that employ the principles of natural selection through such mechanisms as selection, mutation and reproduction. Feuz and Cook [5] succeeded in implementing the first context-aware system based on machine learning technique using transfer learning mechanism from evolutionary computing to track users with Alzheimer disease. However, their work could not differentiate many but different activity contexts, could not handle concurrent activities and inherited the limitations of evolutionary computing.
Sachdeva et al. [6] outlined a context-aware recommender system that presented an empirical analysis of the genetic algorithm, decision tree, bagging and boosting in designing a context-aware reasoning engine. They concluded that the genetic algorithm has the highest accuracy.

Generally, evolutionary algorithms have adverse effects on mobile devices and therefore cannot be implemented as mobile applications [7]. For instance, deep learning and genetic algorithms are computational-intensive algorithms that involve a cascade of many layers of nonlinear processing units for extracting and transforming features. To get maximum performance, recurrent neural networks are usually used [8]. They are, therefore, not desirable for mobile devices with limited processing capability. Though evolutionary computing has been successful in areas like structural analysis yet it faces challenges with continuous parametric optimization which involves highly performing numerical methods [9], [10].

The most remarkable area evolutionary computing has received attention is that of multi-objective optimization [11] which requires a small portion of computing time. This means that evolutionary computing applies to problems where multiple solutions are required and where no appropriate method is available. However, there is no guarantee for finding optimal solutions in a finite amount of time [11], [12]. Unfortunately, researchers are not able to determine which evolutionary computing method is better for which problem [12]. This means evolutionary computing methods could not be used in this study where the focus was on classification problems in mobile devices.

Researchers, therefore, concentrated on using Artificial Neural Networks (ANNs) to develop context-aware applications for mobile devices. The common mechanisms for ANN implementations employed by researchers are propagation algorithms [13], scaled conjugate gradient algorithm [14], latent factorization mechanisms based on deep learning techniques [8], [15], [16]. The work of Opoku and Rao [17] enumerated some major limitations associated with some of these mechanisms. Among them was the complex nature of these mechanisms which required extensive computing power and memory that cannot be offered by the current mobile devices. They recommended that the learning algorithms and architectures of ANN have to be controlled. This study used a multi-layer perceptron 
with a sigmoid activation function instead of a recurrent network in a supervised environment using the gradient descent mechanism of the back-propagation algorithm for training due to its simplicity and adaptability [18] with a constant learning rate. A stochastic model of learning, instead of a batch model of learning, with a constant learning rate, was employed to prevent the network from being trapped in local minima. Thus, momentum and adaptive learning rate which perform the same function were ignored in this study. The remaining parts of the paper are divided into three sections. The second section focuses on how data was acquired and processed for the study. The third section looks at the ANN context-aware reason engine modelling and implementation whilst the final section discusses the findings and concludes the paper.

\section{DATA ACQUisition AND PROCESSING}

Research works have indicated that users' preferences differ [19]-[22]. Users tend to rank concurrent activities and select their preferred activity. According to Burke et al. in [23], accurate ranking is obtained with not more than five choices. Thus, the implementation of the context-aware reasoning engine focused on a maximum of five concurrent activities of which the user is to choose the preferred one. Thus there were five input neurons and five output neurons. At any particular time, each of the neurons can assume a value of 0 or 1 but not both. When the value is 0 , it indicates the absence of a category of an activity context otherwise, the neuron assumes a value of 1 . To enhance the flexibility of the network, a bias neuron with a value of 1 is introduced in the input layer and the hidden layer [24]. The total number of weights or interconnections defines the complexity of the network. Let $n$ be the number of neurons in the hidden layer. The total number of interconnections between the input layer and the hidden layer is $6 n$ whereas the number of interconnections between the hidden layer and the output layer is $5(n+1)$. Thus the total number of interconnections or weights is $6 n+5(n+1)$ and is simplified as $11 n+5$. The study therefore aimed at determining the minimum value for $n$ such that at least a $95 \%$ accuracy level is achieved.

Data is needed to train the ANN model. The study used the stochastic learning approach which requires that the data for training the ANN handles all situations but with no repetition of records or data sets. A questionnaire was given to 980 randomly selected respondents to determine how they would select an activity from ranked activities. The completed questionnaire was normalized by using zeros and ones which resulted in 32 unique records as depicted in Table I. A value of one (1) indicates that a category of activity is available for ranking and selection, otherwise, the value is zero (0). Table I shows the input and output values.

From Table I, a row shows the category of activities that are available for prediction at a particular time. The input data for each row can have as many 1's and 0's. However, the corresponding output data can only have 1 and four 0 's except the $32^{\text {nd }}$ row which has no category of activity to predict. This indicates that if the category of activities does not include any of the user's preferences, the reasoning engine should not predict any activity. Where this 1 is placed on every row depends on the ranks of activities on the row. The above data was used to train the ANN. A cycle of training is completed when the entire 32 sets of data have been used in the training. The accuracy level of the training was set to at least $95 \%$.

\begin{tabular}{|c|c|c|c|c|c|c|c|c|c|c|}
\hline \multirow{2}{*}{$\begin{array}{l}\text { Serial } \\
\text { Nos }\end{array}$} & \multicolumn{5}{|c|}{ Input Data } & \multicolumn{5}{|c|}{ Target or Output Data } \\
\hline & $\mathrm{X}_{1}$ & $\mathrm{X}_{2}$ & $\mathrm{X}_{3}$ & $\mathrm{X}_{4}$ & $\mathrm{X}_{5}$ & $\mathrm{Y}_{1}$ & $\mathrm{Y}_{2}$ & $\mathrm{Y}_{3}$ & $\mathrm{Y}_{4}$ & $Y_{5}$ \\
\hline 1 & 1 & 1 & 1 & 1 & 1 & 1 & 0 & 0 & 0 & 0 \\
\hline 2 & 1 & 1 & 1 & 1 & 0 & 1 & 0 & 0 & 0 & 0 \\
\hline 3 & 1 & 1 & 1 & 0 & 1 & 1 & 0 & 0 & 0 & 0 \\
\hline 4 & 1 & 1 & 1 & 0 & 0 & 1 & 0 & 0 & 0 & 0 \\
\hline 5 & 1 & 1 & 0 & 1 & 1 & 1 & 0 & 0 & 0 & 0 \\
\hline 6 & 1 & 1 & 0 & 1 & 0 & 1 & 0 & 0 & 0 & 0 \\
\hline 7 & 1 & 1 & 0 & 0 & 1 & 1 & 0 & 0 & 0 & 0 \\
\hline 8 & 1 & 1 & 0 & 0 & 0 & 1 & 0 & 0 & 0 & 0 \\
\hline 9 & 1 & 0 & 1 & 1 & 1 & 1 & 0 & 0 & 0 & 0 \\
\hline 10 & 1 & 0 & 1 & 1 & 0 & 1 & 0 & 0 & 0 & 0 \\
\hline 11 & 1 & 0 & 1 & 0 & 1 & 1 & 0 & 0 & 0 & 0 \\
\hline 12 & 1 & 0 & 1 & 0 & 0 & 1 & 0 & 0 & 0 & 0 \\
\hline 13 & 1 & 0 & 0 & 1 & 1 & 1 & 0 & 0 & 0 & 0 \\
\hline 14 & 1 & 0 & 0 & 1 & 0 & 1 & 0 & 0 & 0 & 0 \\
\hline 15 & 1 & 0 & 0 & 0 & 1 & 1 & 0 & 0 & 0 & 0 \\
\hline 16 & 1 & 0 & 0 & 0 & 0 & 1 & 0 & 0 & 0 & 0 \\
\hline 17 & 0 & 1 & 1 & 1 & 1 & 0 & 1 & 0 & 0 & 0 \\
\hline 18 & 0 & 1 & 1 & 1 & 0 & 0 & 1 & 0 & 0 & 0 \\
\hline 19 & 0 & 1 & 1 & 0 & 1 & 0 & 1 & 0 & 0 & 0 \\
\hline 20 & 0 & 1 & 1 & 0 & 0 & 0 & 1 & 0 & 0 & 0 \\
\hline 21 & 0 & 1 & 0 & 1 & 1 & 0 & 1 & 0 & 0 & 0 \\
\hline 22 & 0 & 1 & 0 & 1 & 0 & 0 & 1 & 0 & 0 & 0 \\
\hline 23 & 0 & 1 & 0 & 0 & 1 & 0 & 1 & 0 & 0 & 0 \\
\hline 24 & 0 & 1 & 0 & 0 & 0 & 0 & 1 & 0 & 0 & 0 \\
\hline 25 & 0 & 0 & 1 & 1 & 1 & 0 & 0 & 1 & 0 & 0 \\
\hline 26 & 0 & 0 & 1 & 1 & 0 & 0 & 0 & 1 & 0 & 0 \\
\hline 27 & 0 & 0 & 1 & 0 & 1 & 0 & 0 & 1 & 0 & 0 \\
\hline 28 & 0 & 0 & 1 & 0 & 0 & 0 & 0 & 1 & 0 & 0 \\
\hline 29 & 0 & 0 & 0 & 1 & 1 & 0 & 0 & 0 & 1 & 0 \\
\hline 30 & 0 & 0 & 0 & 1 & 0 & 0 & 0 & 0 & 1 & 0 \\
\hline 31 & 0 & 0 & 0 & 0 & 1 & 0 & 0 & 0 & 0 & 1 \\
\hline 32 & 0 & 0 & 0 & 0 & 0 & 0 & 0 & 0 & 0 & 0 \\
\hline
\end{tabular}

In any machine learning modelling task, certain conditions need to be met to have an effective system. The first condition required that duplication of data is avoided in a stochastic model of machine learning [25]. The data set consisted of 32 unique records for training and testing. All rows and columns were thoroughly checked to make sure that there were no missing values, no duplication of data, no typographical error and no transposition error.

The second condition is to prevent multi-collinearity such that the independent variables do not depend on themselves. However, the independent variables should relate to the dependent variables to develop a good model. Correlation matrices were developed to determine the relationship between the variables using Pearson's correlation coefficient, Spearman's correlation coefficient and Kendall's Tau-b. Pearson's correlation coefficient is a parametric test that determines the relationship between two continuous variables whereas both Spearman's correlation coefficient and Kendall's Tau-b are nonparametric. Spearman's correlation coefficient determines the relationship between ranked variables whilst Kendall's Tau-b measures the strength between two variables on an ordinal scale. Since the nature of the category of activities is not clearly defined, all three tests were used such that they should all produce the same interpretation for the data to be used for developing the ANN model. This makes the model a universal solution for the problem.

Statistical Package for Social Sciences (SPSS) software was used to determine the values of the three tests. A 
significance level of 0.05 was used to test the null hypothesis, $\mathrm{H}_{0}$, there is no relationship between the variables. The independent variables correspond to the five ranked activities from the user whereas, the dependent variables correspond to the five categories of activities in which the ANN reasoning engine only predicts one of them. Thus, there were ten variables. Table II shows the results obtained during the test to determine the relationships between the independent variables or users' choices (represented in the table as IV). The statistical value (represented in the table as SV) for the various tests alongside their $\mathrm{p}$-values (also represented in the table as $\mathrm{p}-\mathrm{V})$.

TABLE II: RELATIONSHIP BETWEEN THE INDEPENDENT VARIABLES

\begin{tabular}{|c|c|c|c|c|c|c|c|}
\hline \multirow{2}{*}{ IV } & \multirow{2}{*}{\multicolumn{2}{|c|}{ Test Performed }} & \multicolumn{5}{|c|}{ IV } \\
\hline & & & $1^{\mathrm{st}}$ & \multirow{2}{*}{$\frac{2^{\text {nd }}}{-0.03}$} & \multirow{2}{*}{$\frac{3^{\text {rd }}}{-0.03}$} & \multirow{2}{*}{$\begin{array}{l}4^{\text {th }} \\
-0.03\end{array}$} & \multirow{2}{*}{$\begin{array}{l}5^{\text {th }} \\
-0.03\end{array}$} \\
\hline \multirow{6}{*}{$1^{\mathrm{st}}$} & \multirow{2}{*}{ Pearson } & SV & & & & & \\
\hline & & $\mathrm{p}-\mathrm{V}$ & & 0.43 & 0.43 & 0.43 & 0.43 \\
\hline & \multirow{2}{*}{ Spearman } & SV & & -0.03 & -0.03 & -0.03 & -0.03 \\
\hline & & $\mathrm{p}-\mathrm{V}$ & & 0.43 & 0.43 & 0.43 & 0.43 \\
\hline & \multirow{2}{*}{ Kendall } & SV & & -0.03 & -0.03 & -0.03 & -0.03 \\
\hline & & $\mathrm{p}-\mathrm{V}$ & & 0.43 & 0.43 & 0.43 & 0.43 \\
\hline \multirow{6}{*}{$2^{\text {nd }}$} & \multirow{2}{*}{ Pearson } & SV & -0.03 & & -0.03 & -0.03 & -0.03 \\
\hline & & $\mathrm{p}-\mathrm{V}$ & 0.43 & & 0.43 & 0.43 & 0.43 \\
\hline & \multirow{2}{*}{ Spearman } & SV & -0.03 & & -0.03 & -0.03 & -0.03 \\
\hline & & $\mathrm{p}-\mathrm{V}$ & 0.43 & & 0.43 & 0.43 & 0.43 \\
\hline & & SV & -0.03 & & -0.03 & -0.03 & -0.03 \\
\hline & & $\mathrm{p}-\mathrm{V}$ & 0.43 & & 0.43 & 0.43 & 0.43 \\
\hline \multirow{6}{*}{$3^{\text {rd }}$} & \multirow{2}{*}{ Pearson } & SV & -0.03 & -0.03 & & -0.03 & -0.03 \\
\hline & & $\mathrm{p}-\mathrm{V}$ & 0.43 & 0.43 & & 0.43 & 0.43 \\
\hline & \multirow{2}{*}{ Spearman } & SV & -0.03 & -0.03 & & -0.03 & -0.03 \\
\hline & & $\mathrm{p}-\mathrm{V}$ & 0.43 & 0.43 & & 0.43 & 0.43 \\
\hline & \multirow{2}{*}{ Kendall } & SV & -0.03 & -0.03 & & -0.03 & -0.03 \\
\hline & & $\mathrm{p}-\mathrm{V}$ & 0.43 & 0.43 & & 0.43 & 0.43 \\
\hline \multirow{6}{*}{$4^{\text {th }}$} & \multirow{2}{*}{ Pearson } & SV & -0.03 & -0.03 & -0.03 & & -0.03 \\
\hline & & $\mathrm{p}-\mathrm{V}$ & 0.43 & 0.43 & 0.43 & & 0.43 \\
\hline & \multirow{2}{*}{ Spearman } & SV & -0.03 & -0.03 & -0.03 & & -0.03 \\
\hline & & $\mathrm{p}-\mathrm{V}$ & 0.43 & 0.43 & 0.43 & & 0.43 \\
\hline & \multirow{2}{*}{ Kendall } & SV & -0.03 & -0.03 & -0.03 & & -0.03 \\
\hline & & $\mathrm{p}-\mathrm{V}$ & 0.43 & 0.43 & 0.43 & & 0.43 \\
\hline \multirow{6}{*}{$5^{\text {th }}$} & \multirow{2}{*}{ Pearson } & SV & -0.03 & -0.03 & -0.03 & -0.03 & \\
\hline & & $\mathrm{p}-\mathrm{V}$ & 0.43 & 0.43 & 0.43 & 0.43 & \\
\hline & \multirow{2}{*}{ Spearman } & SV & -0.03 & -0.03 & -0.03 & -0.03 & \\
\hline & & $\mathrm{p}-\mathrm{V}$ & 0.43 & 0.43 & 0.43 & 0.43 & \\
\hline & \multirow{2}{*}{ Kendall } & SV & -0.03 & -0.03 & -0.03 & -0.03 & \\
\hline & & $\mathrm{p}-\mathrm{V}$ & 0.43 & 0.43 & 0.43 & 0.43 & \\
\hline
\end{tabular}

A general observation of Table II shows that the statistical values $(-0.03)$ and the $p$-values $(0.43)$ are the same across the three tests performed. The black-shaded areas are the intersection between the same independent variables. These correlations were ignored since the correlations between different variables were studied. Linking the black-shaded boxes creates a diagonal in the correlation matrix. The diagonal shape divides the matrix into two portions. A critical look at the upper portion of the matrix reveals that it is symmetrical to the lower portion of the matrix. This indicates that the values in the lower portion of the matrix are the same as the upper portion of the matrix. All the p-values in the matrix are greater than 0.05 , the significance level. This indicates that there is not enough evidence to reject the null hypothesis. Accepting the null hypothesis $\left(\mathbf{H}_{0}\right.$ : there is no relationship between the variables) implies that there is no relationship or correlation between the independent variables. The result is very positive for training the neural network since there is no collinearity. However, there should exist a correlation between the independent variables (IV) and the dependent variables which are the ANN reasoning engine predictions (DV) to achieve a better prediction model.

Table III shows the results obtained during the test to determine the relationships between the IVs and DVs. The statistical values (represented in the table as SV) for the various tests alongside their $\mathrm{p}$-values (also represented in the table as $\mathrm{p}-\mathrm{V}$ ) are displayed.

TABLE III: RELATIONSHIP BETWEEN IV'S AND DV'S

\begin{tabular}{|c|c|c|c|c|c|c|c|}
\hline \multirow{2}{*}{ IV } & \multirow{2}{*}{\multicolumn{2}{|c|}{ Test Performed }} & \multicolumn{5}{|c|}{ DV } \\
\hline & & & $1^{\mathrm{st}}$ & $2^{\text {nd }}$ & $3^{\text {rd }}$ & $4^{\text {th }}$ & $5^{\text {th }}$ \\
\hline \multirow{6}{*}{$1^{\text {st }}$} & \multirow{2}{*}{ Pearson } & SV & 1.00 & -0.61 & -0.40 & -0.27 & -0.19 \\
\hline & & $\mathrm{p}-\mathrm{V}$ & 0.00 & 0.00 & 0.01 & 0.07 & 0.16 \\
\hline & \multirow{2}{*}{ Spearman } & SV & 1.00 & -0.61 & -0.4 & -0.27 & -0.19 \\
\hline & & $\mathrm{p}-\mathrm{V}$ & 0.00 & 0.00 & 0.01 & 0.07 & 0.16 \\
\hline & \multirow{2}{*}{ Kendall } & SV & 1.00 & -0.61 & -0.40 & -0.27 & -0.19 \\
\hline & & $\mathrm{p}-\mathrm{V}$ & 0.00 & 0.00 & 0.02 & 0.07 & 0.15 \\
\hline \multirow{6}{*}{$2^{\text {nd }}$} & \multirow{2}{*}{ Pearson } & SV & -0.03 & 0.57 & -0.40 & -0.27 & -0.19 \\
\hline & & $\mathrm{p}-\mathrm{V}$ & 0.43 & 0.00 & 0.01 & 0.07 & 0.16 \\
\hline & \multirow{2}{*}{ Spearman } & SV & -0.03 & 0.57 & -0.40 & -0.27 & -0.19 \\
\hline & & $\mathrm{p}-\mathrm{V}$ & 0.43 & 0.00 & 0.01 & 0.07 & 0.16 \\
\hline & \multirow{2}{*}{ Kendall } & SV & -0.03 & 0.57 & -0.40 & -0.27 & -0.19 \\
\hline & & $\mathrm{p}-\mathrm{V}$ & 0.43 & 0.00 & 0.01 & 0.07 & 0.15 \\
\hline \multirow{6}{*}{$3^{\text {rd }}$} & \multirow{2}{*}{ Pearson } & SV & -0.03 & -0.02 & 0.37 & -0.27 & -0.19 \\
\hline & & $\mathrm{p}-\mathrm{V}$ & 0.43 & 0.46 & 0.02 & 0.07 & 0.15 \\
\hline & \multirow{2}{*}{ Spearman } & SV & -0.03 & -0.02 & 0.37 & -0.27 & -0.19 \\
\hline & & $\mathrm{p}-\mathrm{V}$ & 0.43 & 0.46 & 0.03 & 0.07 & 0.16 \\
\hline & \multirow{2}{*}{ Kendall } & SV & -0.03 & -0.02 & -0.37 & -0.27 & -0.19 \\
\hline & & $\mathrm{p}-\mathrm{V}$ & 0.43 & 0.43 & 0.02 & 0.07 & 0.15 \\
\hline \multirow{6}{*}{$4^{\text {th }}$} & \multirow{2}{*}{ Pearson } & SV & -0.03 & -0.02 & -0.01 & 0.25 & -0.19 \\
\hline & & $\mathrm{p}-\mathrm{V}$ & 0.43 & 0.46 & 0.47 & -0.08 & 0.16 \\
\hline & \multirow{2}{*}{ Spearman } & SV & -0.03 & -0.02 & -0.01 & 0.25 & -0.19 \\
\hline & & $\mathrm{p}-\mathrm{V}$ & 0.43 & 0.46 & 0.47 & -0.08 & 0.16 \\
\hline & \multirow{2}{*}{ Kendall } & SV & -0.03 & -0.02 & -0.01 & 0.25 & -0.19 \\
\hline & & $\mathrm{p}-\mathrm{V}$ & 0.43 & 0.46 & 0.47 & -0.08 & 0.15 \\
\hline \multirow{6}{*}{$5^{\text {th }}$} & \multirow{2}{*}{ Pearson } & SV & -0.03 & -0.02 & -0.01 & -0.01 & 0.18 \\
\hline & & $\mathrm{p}-\mathrm{V}$ & 0.43 & 0.46 & 0.47 & 0.48 & 0.17 \\
\hline & \multirow{2}{*}{ Spearman } & SV & -0.03 & -0.02 & -0.01 & -0.01 & 0.18 \\
\hline & & $\mathrm{p}-\mathrm{V}$ & 0.43 & 0.46 & 0.47 & 0.48 & 0.17 \\
\hline & \multirow{2}{*}{ Kendall } & SV & -0.03 & -0.02 & -0.01 & -0.01 & 0.18 \\
\hline & & $\mathrm{p}-\mathrm{V}$ & 0.43 & 0.46 & 0.47 & 0.48 & 0.17 \\
\hline
\end{tabular}

From Table III, the first choice of IV correlates with the first choice of DV $(r=1.000, p$-value $=0.000 ; \rho=1.000, p$ value $=0.000$ and Kendall's Tau-b $=1.000, p$-value $=0.000$ ), with the second choice of DV $(r=-0.609 p$-value $=0.000 ; \rho$ $=-0.609, \mathrm{p}$-value $=0.0000$ and Kendall's Tau-b $=-0.609, \mathrm{p}-$ value $=0.000)$, and the third choice of DV $(r=-0.398 p$-value $=0.013 ; \rho=-0.398, p$-value $=0.013$ and Kendall's Tau- $b=$ 0.398, p-value $=0.013$ ). This indicates that the first independent variable has effect on the predictions of the first, second and third dependent variables.

The second choice of IV correlates with the second choice of DV $(r=0.571 \mathrm{p}$-value $=0.000 ; \rho=0.571$, $\mathrm{p}$-value $=0.0000$ and Kendall's Tau-b $=0.571$, p-value $=0.000$ ) and the third choice of DV $(r=-0.398 p$-value $=0.013 ; \rho=-0.398$, $p$-value $=0.0013$ and Kendall's Tau-b $=-0.398, \mathrm{p}$-value $=0.015$ ). Thus, the second independent variable influences the predictions of the second and the third dependent variables.

The third choice of users' choice of IV correlates with the third choice of DV $(r=0.373 p$-value $=0.019 ; \rho=0.373$, $\mathrm{p}$ value $=0.0019$ and Kendall's Tau-b $=-0.373$, $\mathrm{p}$-value $=0.021)$. This means that the third independent variable influences the prediction of the third dependent variable only.

The fourth and fifth choices of IV have no correlations with any of the choices of ANN reasoning engine predictions (DV) since the p-values of the fourth and fifth choices' rows are greater than 0.05 . However, the fourth and fifth choices of 
both the users' choice and the ANN reasoning engine predictions are maintained in the data set to train the ANN. This allows for the possibility of users being able to rank and prioritize activities as fourth and fifth choices.

The third condition to be satisfied has to do with the nature of the neural network. The neural network was designed using the backpropagation algorithm based on the gradient descent delta rule with the logistic activation function. The theory of the backpropagation algorithm by Hecht-Nielsen in [26] determined the existence of multiple local minima in network training and the ability of a three-layer backpropagation neural network to approximate any function within the mean square error of the given error when greater than zero. The following actions were taken to satisfy the theory of backpropagation:

- The training error was set to $0.0001>0$ to approximate the function within the mean square error of the given error.

- The study employed a stochastic learning mode instead of a batch learning mode with a constant learning rate and a constant seed number to generate the same random weight values during the initialization of learning parameters for training to help skip the local minima. This ensured that the results obtained during successive training were compared so that the best training yielding at least $95 \%$ testing accuracy was selected.

- A three-layer neural network was chosen for this study with one input layer, one hidden layer unit and one output layer

\section{ANN CONTEXT REASONING ENGINE MODELLING AND IMPLEMENTATION}

Let $\mathrm{X}_{\mathrm{i}}(\mathrm{t})$ represent the input neurons in a discrete-time $\mathrm{t}$, $\forall t, t>0$ and $i \in \mathbb{Z}^{+}$and $0 \leq i \leq 5$. A bias neuron was introduced as $\mathrm{X}_{5}(\mathrm{t})=1, \forall t$. Thus each of the input neurons can have a value of 1 or 0 but not both. The arrangement of the input layer neurons was based on the ranking of the activity data by the user. Regarding the hidden layer, let $\mathrm{H}_{\mathrm{j}}(\mathrm{t})$ represents hidden layer neuron value in a discrete-time $\mathrm{t}$, $\forall t, t>0$, such that $\forall j, 0 \leq j \leq n$ and $j, n \in \mathbb{Z}^{+}$where $\mathrm{n}$ represents the number of neurons in the hidden layer. Also applying a bias neuron in the hidden layer such that $\mathrm{H}_{\mathrm{n}}(\mathrm{t})=1$, $\forall t$. Also, let $\mathrm{Y}_{\mathrm{k}}(\mathrm{t})$ be the target output of the network in a discrete-time $\mathrm{t}, \forall t, t>0$, such that $\forall k, 0 \leq k<5$ and $k \in$ $\mathbb{Z}^{+}$. Thus only one neuron in the output layer should have a value of 1 whilst the others have to be zero. The arrangement of the output layer neurons was based on the ranking of the activity data by the user.

An activation function, sigmoid function $\varnothing(x)$ was used to non-linearize the values of $\mathrm{H}_{\mathrm{j}}(\mathrm{t})$ 's and $\mathrm{Y}_{\mathrm{k}}(\mathrm{t})$ 's. Since all the neurons accept and output either 0 or 1 , the sigmoid function was given as $\emptyset(x)=\frac{1}{1+e^{-\gamma x}}$ where $\gamma$ is a free parameter that controls the shape of the function. For simplicity, $\gamma=1$ was used and hence $\emptyset(x)=\frac{1}{1+e^{-x}}$ There exist weights in which the network training sought to optimise their values so that a solution is obtained for the network. Let $\mathrm{W}_{\mathrm{i}, \mathrm{j}}$ be the weights between the input neurons and the hidden neurons such that $\forall W_{i, j},\left|W_{i, j}\right| \leq 1, \forall i, 0 \leq i \leq 5$ and $\forall j, 0 \leq j \leq n$. Similarly, let $\mathrm{W}_{\mathrm{j}, \mathrm{k}}$ be the weights between the hidden neurons and the output neurons such that $\forall W_{j, k},\left|W_{j, k}\right| \leq 1, \forall j, 0 \leq j \leq n$ and $\forall k, 0 \leq k<5$. The weight values were randomized before the start of the training.

The model for the value of the hidden neurons was obtained by applying the sigmoid function on the weighted inputs that connect the various neurons. Thus the model for obtaining the values of $\mathrm{H}_{\mathrm{j}}(\mathrm{t}), \forall j, 0 \leq j \leq n$ and $\forall t, t>0$ was:

$$
\mathrm{H}_{j}(\mathrm{t})=\frac{1}{1+e^{-\left(\sum_{i=0}^{m} X_{i}(t) \times W_{i, j}(t)\right)}} \in \mathbb{R}^{+}
$$

Similarly, the model for the output neurons value was obtained by applying the sigmoid function on the weighted hidden neuron values that connect the various output neurons. Thus the model for obtaining the values of $\mathrm{Y}_{\mathrm{k}}(\mathrm{t}), \forall k, 0 \leq k<$ 5 and $\forall t, t>0$ was:

$$
\mathrm{Y}_{k}(\mathrm{t})=\frac{1}{1+e^{-\left(\sum_{j=0}^{n} H_{j}(t) \times W_{j, k}(t)\right)}} \in \mathbb{R}^{+}
$$

The values of $\mathrm{H}_{\mathrm{j}}(\mathrm{t})$ 's and $\mathrm{Y}_{\mathrm{k}}(\mathrm{t})$ 's were not rounded to the nearest whole number as done in other research works like Opoku (2013). This ensured that correct weight values were obtained at the end of the training.

Let $\mathrm{E}$ be the error of a neuron whose target output is $\mathrm{T}$ and predicted an output $\mathrm{P}$. Using the square error measure, $E=$ $\frac{1}{2}(T-P)^{2}$. The scalar factor, $\frac{1}{2}$ was introduced for convenience elimination of the scalar multiplication of the results after derivation. From equation (2), $\mathrm{Y}_{k}(\mathrm{t})$, the predicted output is a function of the activation function, $\varnothing(x)$, such that $x$ in $\emptyset(x)$ was replaced with $\sum_{j=0}^{n} H_{j}(t) \times W_{j, k}(t)$ to obtain $\varnothing\left(\sum_{j=0}^{n} H_{j}(t) \times W_{j, k}(t)\right)$, a function of the neurons in a layer. Thus for each neuron, $\mathrm{k}, \forall k>0$, the output was given as $P_{k}=\varnothing\left(n e u_{k}\right)=\varnothing\left(\sum_{j=0}^{n} H_{j} \times W_{j, k}\right)$ where $\emptyset$ is the activation function, $H_{j}$ is the weighted sum of the neurons in the hidden layer with a total number of $n$. At the input layer, $H_{j}$ 's are simply replaced with the inputs $X_{i}{ }^{\prime} s . W_{j, k}$ represents the weight between a hidden neuron, $\mathrm{j}$ and an output neuron $\mathrm{k}$. $\varnothing$ is a non-linear and continuous function. It is therefore differentiable. Given $\emptyset(x)=\frac{1}{1+e^{-x}}$ and applying the quotient rule such that if $y=\frac{U}{V}$ then $\frac{d y}{d x}=\frac{V \frac{d u}{d x}-U \frac{d v}{d x}}{V^{2}}$, the derivation is:

$$
\frac{d \emptyset}{d x}=\frac{0\left(1+e^{-x}\right)-1\left(-e^{-x}\right)}{\left(1+e^{-x}\right)^{2}}=\frac{e^{-x}}{\left(1+e^{-x}\right)^{2}}
$$

The expression can be simplified as:

\[ \frac{d \emptyset}{d x}=\frac{1}{1+e^{-x}}\left(1-\frac{1}{1+e^{-x}}\right) \]
which represents $\frac{d \emptyset}{d x}=\emptyset(x)(1-\emptyset(x))$.

The derivation of the error of the neuron to the weights is obtained by partial derivation using the chain rule mechanism shown below:

$$
\frac{\partial E}{\partial W_{j, k}}=\frac{\partial E}{\partial P_{k}} \times \frac{\partial P_{k}}{\partial n e u_{k}} \times \frac{\partial n e u_{k}}{\partial W_{j, k}}
$$


Evaluating the factors in equation (3), starting with the last factor implies:

$$
\begin{aligned}
& \quad \frac{\partial n e u_{k}}{\partial W_{j, k}}=\frac{\partial}{\partial W_{j, k}}\left(\sum_{j=0}^{n} H_{j} \times W_{j, k}\right)=\frac{\partial}{\partial W_{j, k}}\left(H_{j} \times W_{j, k}\right)= \\
& \boldsymbol{H}_{\boldsymbol{j}}
\end{aligned}
$$

Thus for the input layer, $H_{j}$ becomes $X_{i}$.

Next, the middle factor of equation (3) is evaluated:

$$
\frac{\partial P_{k}}{\partial n e u_{k}}=\varnothing\left(n e u_{k}\right)=\emptyset\left(n e u_{k}\right)\left(1-\emptyset\left(n e u_{k}\right)\right)
$$

The first factor of equation (3) is then evaluated:

$$
\begin{aligned}
& \frac{\partial E}{\partial P_{k}}=\frac{\partial}{\partial P_{k}}\left(\frac{1}{2}(T-P)^{2}\right)=2\left(\frac{1}{2}\right)(-1)(T-P)^{2-1}= \\
& -(T-P)=P-T
\end{aligned}
$$

From equation (3), multiplying the first and the middle factors gives the error for the output neuron. Hence the error for the output neurons, $\mathrm{E}_{\mathrm{k}}, \forall k, 0 \leq k<q$ is given as:

$$
\begin{gathered}
\boldsymbol{E}_{\boldsymbol{k}}=\boldsymbol{P}_{\boldsymbol{k}}\left(\mathbf{1}-\boldsymbol{P}_{k}\right)\left(\boldsymbol{P}_{\boldsymbol{k}}-\boldsymbol{T}_{\boldsymbol{k}}\right) \text { or }-\boldsymbol{E}_{\boldsymbol{k}}=\boldsymbol{P}_{\boldsymbol{k}}(\mathbf{1}- \\
\left.\boldsymbol{P}_{\boldsymbol{k}}\right)\left(\boldsymbol{T}_{\boldsymbol{k}}-\boldsymbol{P}_{\boldsymbol{k}}\right)
\end{gathered}
$$

Similarly, the error in the hidden layer, $E_{\mathrm{j}}$, is computed by considering the neuron $\mathrm{k}, \forall k, 0 \leq k<q$, in the output layer and the neuron $\mathrm{j}, \forall j, 0 \leq j \leq n$, in the hidden layer as $\frac{\partial E\left(H_{j}\right)}{\partial H_{j}}=\frac{\partial}{\partial H_{j}}\left(n e u_{0}, n e u_{1}, n e u_{2}, n e u_{3}, \ldots, n e u_{q-1}\right)$. This evaluated as:

$$
\begin{aligned}
& \frac{\partial E}{\partial H_{j}}=\sum_{k=0}^{q-1}\left(\frac{\partial E}{\partial n e u_{k}} \times \frac{\partial n e u_{k}}{\partial H_{j}}\right)=\sum_{k=0}^{q-1}\left(\frac{\partial E}{\partial P_{k}} \times \frac{\partial P_{k}}{\partial n e u_{k}} \times\right. \\
& \left.W_{j, k}\right)
\end{aligned}
$$

This is finally written as:

$$
E_{j}=H_{j}\left(1-H_{j}\right)\left(\sum_{k=0}^{q-1}\left(E_{k} \times W_{j, k}\right)\right)
$$

From equation (5) the errors of the neurons in the hidden layer can only be computed after the errors in the output layer have been obtained in equation (4). The error of the bias, $E_{b}$, is not used since $\forall E_{b}, E_{b}=0$ given that $H_{b}\left(1-H_{b}\right)=0$. Thus the bias was only used in the forward pass.

In general, the weight, $W_{j, k}$, between neuron $\mathrm{j}$ and neuron $\mathrm{k}$ with a learning rate, $\eta$, is updated as $W_{j, k}^{\text {new }}=W_{j, k}^{\text {old }-}$ $\eta \Delta W_{j, k}$. The negative value of the learning rate updates the weights in the minimum direction but not the maximum direction.

Given that, $\Delta W_{j, k}=\frac{\partial E}{\partial W_{j, k}}$, from equations (3) and (4) above. $\Delta W_{j, k}=H_{j} \times E_{k}$ Thus the output of neuron $\mathrm{j}$ multiplied by the error of neuron $\mathrm{k}$ gives the weight change. Using $-E_{k}=P_{k}\left(1-P_{k}\right)\left(T_{k}-P_{k}\right)$ from equation (4), the weights between the hidden layer and the output layer, $W_{j, k}$, $\forall j, 0 \leq j \leq n$ and $\forall k, 0 \leq k<q$ are obtained as:

$$
W_{j, k}^{n e w}=W_{j, k}^{\text {old }}+\eta H_{j} E_{k}
$$

Similarly, equation (5) gives $-\mathrm{E}_{\mathrm{j}}$ and the weights between the input layer and the hidden layer, $W_{i, j}, \forall i, 0 \leq i \leq m$ and $\forall j, 0 \leq j \leq n$ are obtained as:

$$
W_{i, j}^{\text {new }}=W_{i, j}^{\text {old }}+\eta X_{i} E_{j}
$$

Equations 1 to 7 except 3 were implemented as software that was used to train the neural network. With the above mathematical models, the training data was used to determine the values of $\mathrm{W}_{\mathrm{i}, \mathrm{j}}$ and $\mathrm{W}_{\mathrm{j}, \mathrm{k}}$.

The procedures for training the ANN are outlined below:

00 Begin

01 Initialize the weights

02 Read the first set of training data

03 For iterations $=1$ to an assigned value

04 Determine hidden neurons values

05 Apply sigmoid function on the values of the hidden neurons

06 Determine output neurons values

07 Apply sigmoid function on output neurons values

08 Calculate the error for each output neuron

09 If the error for each output neuron is less than 0.00001

10 Go to Step 19

11 Else

12 Update the weights using the error

13 End if

14 Read the next set of training data

15 If ((No training data) then

16 Read the first set of training data

17 End if

18 Next For

19 Test the model. If not acceptable, adjust the parameters, go to Step 01

20 Stop.

The training was done on a trial-and-error basis by manipulating the values of some of the parameters. The input neurons, the output neurons and the random seed were constant throughout the training. There were five input neurons and five output neurons corresponding to five choices the user make in ranking the activity data. The random seed was introduced with a constant value of 1234 to obtain the same random numbers for the weights during the start of the training. This ensured that the random numbers for the weights remained constant during the start of each training session so that the study was able to concentrate on the other parameters. These parameters were the number of hidden neurons, learning rate and the number of iterations. These were changed on a trial-and-error mechanism until the optimal solutions were obtained with the following parameters: number of hidden neurons 2 , number of iterations 40,000 and learning rate 0.3 . Fig. 1 shows the architecture of the model at the end of the training. 


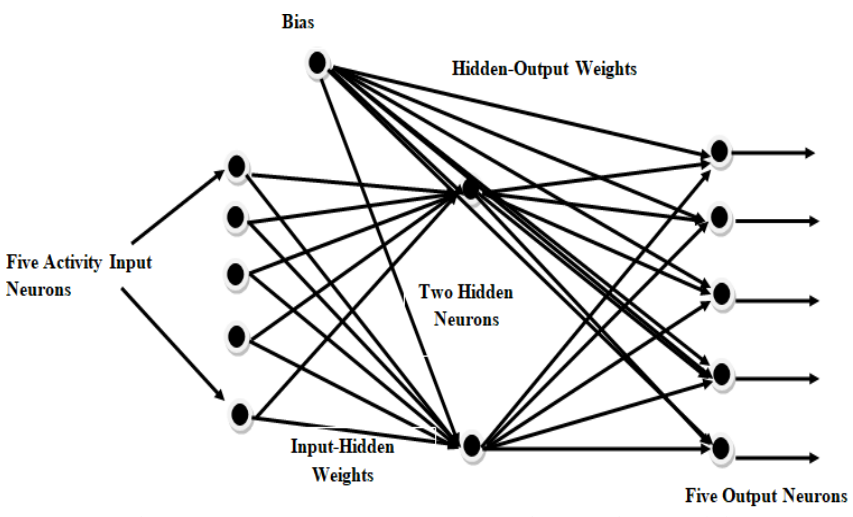

Fig. 1. ANN Context-Aware Reasoning Engine Model.

From Fig. 1, there are five input and output neurons with two neurons in the hidden layer unit. A bias neuron supports the input and hidden neurons. The interconnections or the weights determine the output value of a particular input data. The values of the weights at the end of the model design are displayed in Tables IV and V. These tables respectively represent the weights between input neurons and the hidden neurons (Table IV), and the weights between the hidden neurons and the output neurons (Table V):

TABLE IV: WEIGHT VALUES BETWEEN INPUTS AND HIDDEN NEURONS

\begin{tabular}{ccc}
\hline \multirow{2}{*}{ Input Neurons } & \multicolumn{2}{c}{ Hidden Neurons } \\
\cline { 2 - 3 } & $\mathrm{H}_{1}$ & $\mathrm{H}_{2}$ \\
\hline $\mathrm{X}_{1}$ & 9.9010 & 17.8627 \\
$\mathrm{X}_{2}$ & 7.3004 & -11.2209 \\
$\mathrm{X}_{3}$ & 4.5868 & 8.4912 \\
$\mathrm{X}_{4}$ & -0.0206 & -3.9225 \\
$\mathrm{X}_{5}$ & -0.0005 & 0.2443 \\
Bias & -4.9540 & -0.1060 \\
\hline
\end{tabular}

TABLE V: WEIGHT VALUES BETWEEN HIDDEN AND OUTPUT NEURONS

\begin{tabular}{cccccc}
\hline Hidden & \multicolumn{5}{c}{ Output Neurons } \\
\cline { 2 - 6 } Neurons & Y1 & Y2 & Y3 & Y4 & Y5 \\
\hline $\mathrm{H}_{1}$ & 15.8189 & 10.7734 & -14.4279 & -12.2827 & -30.7500 \\
$\mathrm{H}_{2}$ & 10.1640 & -17.6906 & 27.4679 & -16.7927 & 12.1429 \\
Bias & -20.8825 & -4.3348 & -17.7672 & 4.8777 & -3.4899 \\
\hline
\end{tabular}

There are 27 weights or interconnections in the model. These weights are required by any application that will use the model as a reasoning engine. The code snippet in Fig. 2 below illustrates how to use the weights in the implementation.

\section{DISCUSSIONS AND CONCLUSION}

The study deduced that the number of neurons in a hidden layer does not solely affect the accuracy level of a neural network. However, the accuracy of a network depends also on the type of learning algorithm, the learning parameters and the number of epochs for training. Training a network without approximation of neuron's output to the nearest whole number increases the accuracy level of the network at the end of the training. The number of iterations after training does not affect the network. The findings partially confirmed Larose's work in [27] which indicated that too many neurons slow down training and also partially contradicts a portion of that concluded that too many neurons result in higher accuracy. This study refines the contradictory portion by stating that an appropriate number of neurons results in higher accuracy.

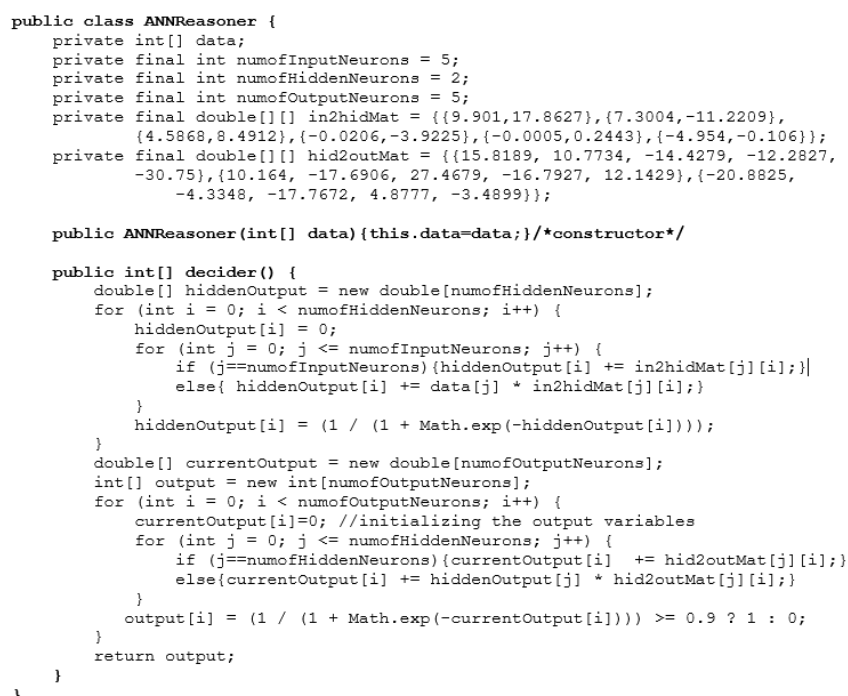

Fig. 2. Listing I: ANNCRE Implementation Class in Java.

The findings confirmed the works of Karosliya in [28] and Heaton in [24]. They asserted that there are so many factors involved in training a network and thus, following specific principles are not always applicable. Training should be done by trial-and-error mechanisms. This study also expands their work by stating that the best training should give an optimal number of weights to minimize the total number of interconnections and processing power. The findings extend the work of Hassan and Hamada in [29]. They argued that a training process will converge to a solution in a finite number of iterations through adaptive learning rate and momentum mechanisms. However, the study employed a stochastic backpropagation algorithm which also performs the functions of adaptive learning rate and momentum. The findings contradict the work of Opoku in [30] which argued that to obtain a high level of accuracy in network training, approximate the outputs to the nearest whole number when dealing with whole numbers. The study found out that approximating the hidden neuron values during training usually leads to underfitting or overfitting.

Training and implementing a neural network requires a lot of time. Researchers should weigh all the possible outcomes on trial-and-error bases such as the number of iterations, learning algorithm, learning parameters and network size; and determine the appropriate parameters that yield the best results that minimize interconnections, storage capacity and processing power since computing resources are scarce.

\section{REFERENCES}

[1] S. K. Opoku. Activity Context Modelling in Context-Aware Environment. International Journal of Computer Applications Technology and Research, vol. 5, no. 11, pp. 687-692, 2016.

[2] A. K. Dey. Context-aware computing. In Ubiquitous Computing Fundamentals, 2010.

[3] N. Naeem, R. Bashroush, R. Anthony, M. A. Azam, A. R. Tawil and S. E. A. Wee. Activities of daily life recognition using process representation modelling to support intention analysis. International Journal of Pervasive Computing and Communications, vol. 1, no. 3, pp. 347-371, 2015.

[4] M. Sharif and A. A. Alesheikh. Context-aware movement analytics: implications, taxonomy and design framework. Wiley Interdisciplinary Reviews: Data mining and knowledge discovery, vol. 8, no. 1, p. 1233, 2018.

[5] D. K. Feuz and D. J. Cook. Heterogeneous transfer learning for activity recognition using heuristic search techniques. International 
Journal of Pervasive Computing and Communications, vol. 10, no. 4, pp. 393-418, 2014.

[6] N. Schdeva, R. Dhir and A. Kumar. Empirical analysis of machine learning techniques for context aware recommender systems in the environment of IoT. In International conference on advances in information communication technology and computing, 2016.

[7] M. D. C. Rodriguez-Hernandez and S. Ilarri. Research contextaware recommendation systems in mobile environment. Jornada de Jovenes Investigadores del 13A, vol. 2, pp. 59-70, 2017.

[8] J. Lian, F. Zhang, X. Xie and G. Sun. CCCFNet: A content-boosted collaborative filtering neural network for cross domain recommender systems. In 26th International Conference on World Wide Web Companion, 2017.

[9] Z. Farhoudi and S. Setayeshi. Fusion of deep learning features with mixture of brain emotional learning for audio-visual emotion recognition. Speech Communication, vol. 127, pp. 92-103, 2020.

[10] J. Zhao, W. Wang, Z. Zhang, Q. Sun and H. Q. Huo. TrustTF: A tensor factorization model using user trust and implicit feedback for context-aware recommender systems. Knowledge-Based Systems, vol. 209, p. 106434, 2020.

[11] K. Deb. Multi-objective optimization using evolutionary algorithms. New York: John Wiley \& Sons, 2001.

[12] G. Narzisi. Evolutionary algorithms: a short introduction. 2008.

[13] H. D. Mehr, H. Polat and A. Cetin. Resident activity recognition in smart homes by using artificial neural networks. In 4th international conference on smart grid congress and fair, Istanbul, 2016.

[14] Y. A. Salam. A proactive multi-type context-aware recommender system in the environment of internet of things. 2016.

[15] H. Jing and A. J. Smola. Neural survival recommender. In Tenth ACM International Conference on Web Search and Data Mining, 2017.

[16] A. Martin, P. Zarate and G. Camillieri. A multi-criteria recommender system based on users' profile management. In Multiple Criteria Decision Making, Springer International Publishing, pp. 83-89, 2017.

[17] S. K. Opoku and D. Subba Rao. A robust mechanism for artificial neural network context-aware recommender systems in mobile environment. International Journal of Computer Science and Information Technology Research, vol. 5, no. 3, pp. 53-60, July 2017.

[18] A. Quesada. 5 algorithms to train a neural network. 30 April 2017. [Online]. Available: https://www.neuraldesigner.com/blog/5_algorithms_to_train_a_ne ural_network.

[19] S. K. Opoku and S. Appiah. Automating students' activities in Higher Educational Institutions. International Journal of Computer Applications Technology and Research, vol. 5, no. 11, pp. 693-697, 2016.

[20] M. Karimi, D. Jannach and M. Jugovac. News recommender system - Survey and roads ahead. Information Processing and Management, 2018.

[21] J. Auda, D. Weber, A. Voit and S. Schneegass. Understanding user preferences towards rule-based notification deferral. In $\mathrm{CHI}$ Conference on Human Factors in Computing Systems, 2018.

[22] F. Li, F. Shuang, Z. Liu and X. Qian. A cost-constrained video quality satisfaction study on mobile devices. IEEE Transactions on Multimedia, vol. 20, no. 5, pp. 1154-1168, 2018.

[23] A. Burke, J. Smyth and K. Olson. The effect of ranking questions format on data quality in mail surveys. In MAPOR conference, Chicago, II, 2015.

[24] J. Heaton. Introduction to neural network for Java. 2nd ed., USA: Heaton Research, 2016.

[25] E. Alcobaça, S. M. Mastelini, T. Botari, B. A. Pimentel, D. R. Cassar, A. C. P. De Leon Ferreira and E. D. Zanotto. Explainable Machine Learning Algorithms for Predicting Glass Transition Temperatures. Acta Materialia, vol. 188, pp. 92-100, 2020.

[26] R. Hecht-Nielsen. Theory of the backpropagation neural network. Neural networks for perception, pp. 65-93, 1992.

[27] D. T. Larose. Discovering knowledge in data: An introduction to data mining. Wiley-Interscience, 2005.

[28] S. Karosliya. Approximating number of hidden layer neurons in multiple hidden layer BPNN architecture. International Journal of
Engineering Trends and Technology, vol. 31, no. 6, pp. 714-717, 2012.

[29] M. Hassan and M. Hamada. Performance comparison of featured neural network trained with back-propagation and delta rule techniques for movie rating prediction in multi-criteria recommender systems," Informatica, vol. 40, no. 4, p. 409, 2016.

[30] S. K. Opoku. Agent-based automated negotiation system for handling vague data using artificial neural network with adaptive backpropagation algorithm. International Journal of Advances in Computer Science and Technology, vol. 2, no. 11, pp. 247-255, 2013. 